\title{
NLS-dependent and insufficient nuclear localization of XAGE-1 splice variants
}

\author{
RUIHUA ZHAO ${ }^{1}$, BIKUI TANG ${ }^{1}$, YANG LIU ${ }^{1,2}$ and NAISHUO ZHU ${ }^{1}$ \\ ${ }^{1}$ Laboratory of Molecular Immunology, Department of Microbiology, State Key Laboratory of Genetic Engineering, \\ School of Life Science, Institute of Biomedical Science, Fudan University, Shanghai 200433; \\ ${ }^{2}$ Shanghai Institute of Quality Inspection and Technical Research, Shanghai 200233, P.R. China
}

Received November 22, 2010; Accepted December 29, 2010

DOI: $10.3892 /$ or.2011.1175

\begin{abstract}
XAGE-1 is a member of the cancer/testis antigen family and it was first identified by searching for the PAGE/ GAGE-related genes. Four transcript variants XAGE- $1 a,-1 b$, $-1 c$ and $X A G E-1 d$ have been discovered and have a broad expression in cancer. As a prominent transcript, XAGE-1b is encoded by 81 amino acids with a molecular weight of $9 \mathrm{kDa}$. We determined the cellular localization of all four splice variants by confocal microscopy analysis. Among these, XAGE-1a, -1b and -1c showed distinct speckled nuclear localization, while XAGE-1d was distributed evenly both in the cytoplasm and nucleus. By deletion mutagenesis and site directed mutagenesis, we identified the bipartite nuclear localization signal and found that it contributes to the nuclear localization of XAGE-1 variants; but the nuclear localization signal (NLS) only cannot form the characterized distribution of XAGE-1b; amino acids 25-42 also play a role in the formation of nuclear speckles. XAGE-1b, the main transcript of XAGE-1, may act as a partner protein or a member of a protein complex, which plays a role in tumor development and progression.
\end{abstract}

\section{Introduction}

$X A G E-1$ (X antigen family, member 1) was discovered by homology walking strategy searching the expressed sequence tag (EST) database (1), it is located on chromosome Xp11.21-p11.22 (2,3). The expression profile defined XAGE-1 as one member of cancer/testis antigen. Based on the position of encoded gene on the chromosome, it was categorized into CT-X antigens (4).

Correspondence to: Dr Naishuo Zhu, Laboratory of Molecular Immunology, Department of Microbiology, State Key Laboratory of Genetic Engineering, School of Life Science, Institute of Biomedical Science, Fudan University, Shanghai 200433, P.R. China

E-mail: nzhu@fudan.edu.cn

Key words: $\mathrm{X}$ antigen family member 1, cellular localization, nuclear localization signal
There are $44 \mathrm{CT}$ (cancer/testis) antigen families discovered currently (5). Every CT gene family may contain multiple members or splice variants. The first CT antigen gene MAGE1 (melanoma-associated antigen 1) was identified in melanoma cell line by autologous typing (6), 12 members clustered at Xq28 have been found in this family (7). Owing to their highly restricted expression patterns in normal tissues and their expression in a wide range of human tumor types, CT antigens have been regarded as ideal targets for cancer vaccines (4).

$X A G E-1$ consists of 4 alternative splice variants: $X A G E-1 a$, $-1 b,-1 c$ and $-1 d(3,8)$. It expressed mainly restricted to testis, placenta and brain in normal tissues, while widely expressed in tumors, such as in: Ewing's sarcoma, alveolar rhabdomyosarcomas, lung adenocarcinoma, prostate cancer, and melanoma (1-3,9-11). As a prominent transcript, XAGE-1b is encoded by 81 amino acids, with molecular weight of $9 \mathrm{kDa}$ $(9,12)$. Coupled expression of GFP with XAGE-1a and -1b showed the distinct speckled distribution in the nucleus (3); $\mathrm{mAb}$ analysis detected XAGE-1b located also in the nucleus (12). In this study, we have described the cellular distribution of the all four XAGE-1 variants and detected their corresponding protein expression, explored the mechanisms for the cellular distribution and the minimum sequence for the characterized nuclear localization.

\section{Material and methods}

Cell culture and transfection. COS7 and 293T cells were cultured in Dulbecco's modified Eagle's medium (DMEM, Invitrogen) supplemented with $10 \%$ fetal calf serum (Hangzhou Sijiqing Biological Engineering Materials Co., Ltd.), 100 U/ml penicillin, and streptomycin $(100 \mu \mathrm{g} / \mathrm{ml}$, Gibco). Cell lines were incubated at $37^{\circ} \mathrm{C}$ in humidified air with $5 \% \mathrm{CO}_{2}$.

Plasmid construct. The four XAGE-1 spliceosomes were amplified from testis library (Takara) by using primers as follows: AF: 5'-TCGACTCGAGATGCTCCTTTGGTGCC CAC-3' and AR: 5'-CCGGAATTCGAACTTGTGGTTGCT CTTCAC-3'; BF: 5'-TCGACTCGAGATGGAGAGCCCCA AAAAGAAGA-3' and AR: as above; CF: 5'-TCGACTCGA GAAGGCCCTCGAAGTCGTCGT-3' and CR: 5'-CCGGAA TTCGAACTTGTGGTTGCTCTTCAC-3'; DF: 5'-CGAC 
A
XAGE-1a
mllwcppqcacsl--gvfpsapspvwg---trrsc------epatrvpevwilspll---
$X A G E-1 b$
$X A G E-1 c$
XAGE-1d
--mrchahgpsclvtaitreeggprsggaqaklgccwgypsprstwnpdrrfwtpqtgpg
- mrchahgpsclvtaitreeggprsggaqaklgccwgypsprst whpdrrfweptgpg
--rhgghtqtqnhtasprspvmespkkknqqlkvgilhlgsrqkkiriqlrsqcatwkvi
XAGE-1a
$X A G E-1 b$
$X A G E-1 C$
$X A G E-1 d$
egrherhtqtqnhtasprspvmespkkknqql kvgilhlgsrqkkiriqlrsqcatwkvi
XAGE -1 a
$X A G E-1 b$
$X A G E-1 c$
$X A G E-1 d$
ckscisqtpginldlgsgvkvkiipkeehckmpeageeqpqv ckscisqtpginldlgsgvkvkiipkeehckmpeageeqpqv ckscisqtpginldlgsgvkvkiipkeehckmpeageeqpqv ------rdmegdl qel hqsntgdksgf-gfrrqgednt--

0

B

XAGE-1b
$11 F$
$25 F$
$31 R$
$37 R$
$40 R$
$46 R$
$52 R$
$11 F 37 R$

C

XAGE-1b

X-1b-M1

X-1b-M2

X-1b-M3
4

PKKKNQQLKVGILHLGSRQKK PAAANQQLKVGILHLGSRQKK PKKKNQQLKVGILHLGSAQAA PAAANQQLKVGILHLGSAQAA

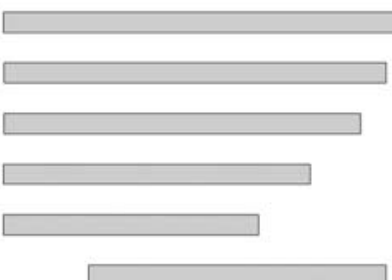

Figure 1. ORF alignment of four XAGE-1 splice variants and schematic representational mutations of XAGE-1b. (A) ORF alignment of four XAGE-1 splice variants, the common sequence is shown; (B) $11 \mathrm{~F}$ represents the N-terminal 10 amino acids deleted mutant; 31R represents the C-terminal 30 amino acids deleted mutant; 11F37R represents both the N-terminal 10 amino acids and C-terminal 30 amino acids deleted; (C) X-1b-M1 represent amino acids 5-7 of XAGE-1b changed from KKK into AAA, X-1b-M2 represents amino acids 21-24 changed from RQKK into AQAA; X-1b-M3 changed both positions. All these ORFs and mutants were cloned into pEGFP-N1 plasmid, and corresponding protein fused to the N-terminal of GFP.

TCGAGATGGAGAGCCCCAAAAAGAAGA-3' and DR: 5'-CCGGAATTCGGGTATTATCTTCACCTTGACG-3'; and were cloned into pEGFP-N1 plasmid. XAGE-1b deletion constructs were generated by PCR amplification using the corresponding region primers, the XhoI and EcoRI sites were used to fuse the ORFs in frame to the pEGFP-N1 plasmid.

Site-directed mutagenesis. The putative NLS point mutations and double-point mutations were generated by using primers XAGE-1bFm: 5'-ATCTCGAGATGGAGAGCCCCGCAG CCGCGAA-3', XAGE-1b-IIaF1: 5'-GCCGCGAACCAGC AGCTGAAAGTCGGGATCCTACA-3', XAGE-1b-IIaF2: 5'-GGATCCTACACCTGGGCAGCGCACAGGCAGCA ATC-3', XAGE-1b-IIaF3: 5'-CAGGCAGCAATCAGGATA CAGCTGAGA-3'; XAGE-1bF: 5'-ATCTCGAGATGGAGAG CCCCAAAAAGAAGAA-3';XAGE-1bR: 5'-CT GAATTCT AACTTGTGGTTGCTCTTCA-3'. Subcloned into pEGFPN1 plasmid. All the clones were confirmed by sequencing.

Cell transfection and confocal microscopy. Cells were seeded onto coverslips in 6-well dishes and incubated overnight to have an approximate confluency of $60-70 \%$. Transfection was performed using Lipofectamine ${ }^{\mathrm{TM}} 2000$ (Invitrogen) according to the manufacturer's instructions. Briefly, diluted Lipofectamine 2000, $2 \mu 1$ in $250 \mu 1$ Opti-MEM medium (Gibco), was incubated for $5 \mathrm{~min}$, mixed with diluted $2 \mu \mathrm{g}$ plasmid the in $250 \mu 1$ Opti-MEM medium, incubated for
$20 \mathrm{~min}$ at room temperature, the added to the cell wells, 4-6 h later the DMEM medium was change to $10 \%$ serum.

Thirty-six hours after transfection, cells were washed in phosphate-buffered saline (PBS, $\mathrm{pH} 7.4$ ), then fixed in $4 \%$ paraformaldehyde for $10 \mathrm{~min}$ on ice, followed by washing in PBS, cells were stained in the dark with $100 \mathrm{ng} / \mathrm{ml}$ DAPI (Sigma) with $0.1 \%$ Triton X-100 for $20 \mathrm{~min}$, cells were finally washed twice in PBS, coverslips were mounted onto glass slides and were sealed with nail polish, imaged by Leica TCS-SP5 confocal microscopy (Germany). Fluorescent cells were counted using Olympus IX51 fluorescent microscope (Japan).

Western blot. After $48 \mathrm{~h}$ of transfection, cells were harvested in tubes, washed with phosphate-buffered saline (PBS, $\mathrm{pH}$ 7.4) two times and then incubated with cell lysis buffer (Shanghai Shenergy Biocolor Bioscience \& Technology Company) on ice for 10-20 min. The lysates were centrifuged at $13000 \mathrm{rpm}$ for $10 \mathrm{~min}$ at $4^{\circ} \mathrm{C}$ and the supernatants were transferred to the new tubes. Supernatants were quantified by Bradford assay, $30 \mu \mathrm{g}$ of total protein were geled by SDS-PAGE and transferred to the nitrocellulose membrane (Millipore). The membrane were blocked with 5\% skim milk in TBST ( $\mathrm{pH} \mathrm{7.4)} \mathrm{for} 3 \mathrm{~h}$ before incubated with rabbit polyclonal GFP antibody (ab290, Abcam plc.) 1:5000 diluted in TBS overnight at $4{ }^{\circ} \mathrm{C}$, washed with TBST four times, then incubated with horseradish peroxidase (HRP) conjugated 


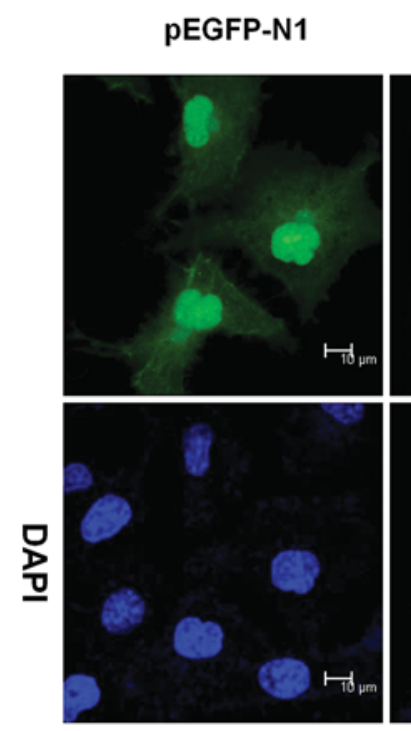

pEGFP-N1-X-1a

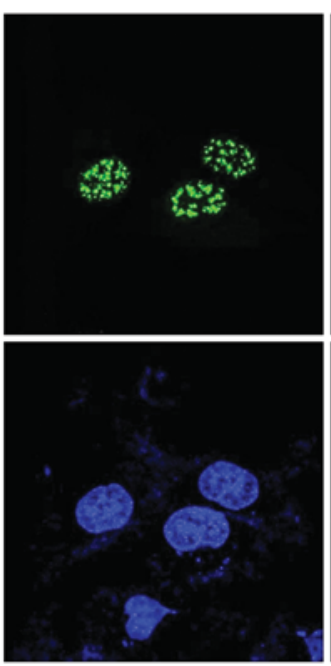

pEGFP-N1-X-1b
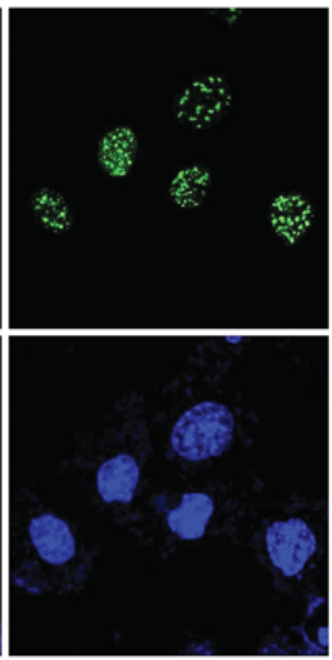

pEGFP-N1-X-1c

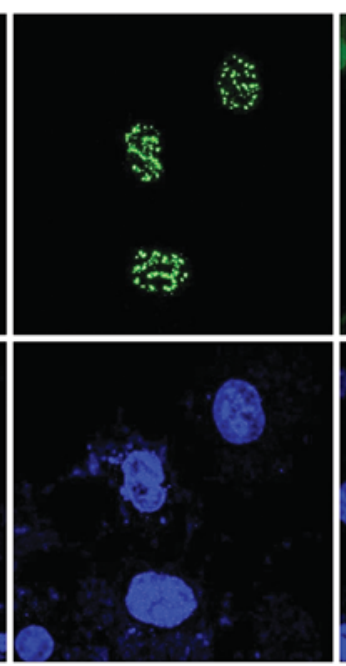

pEGFP-N1-X-1d

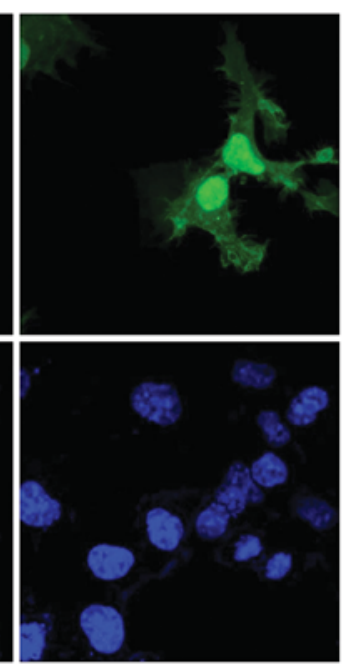

Figure 2. Cellular localization of four XAGE-1 variants in COS7 cells. Fluorescence of GFP, X-1d/GFP is seen both in the cytoplasm and nucleus, while X-1a/GFP, X-1b/GFP, X-1c/GFP localize in the nucleus predominantly and show charateristic dotted distribution. Corresponding nuclei were stained with DAPI (the lower panel). Cells were imaged using Leica TCS SP5 confocal microscope.

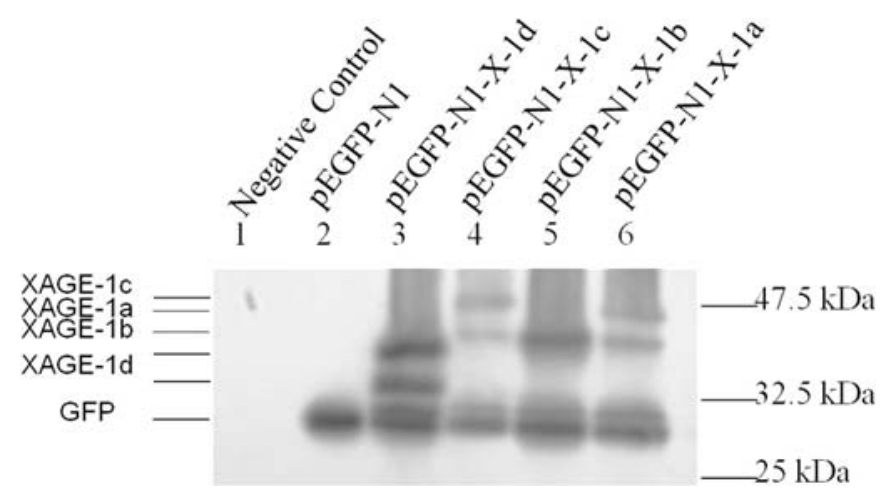

Figure 3. Western blot detection of the protein expression of four XAGE-1 splice variants. COS7 cells were transfected with pEGFP-N1-X-1a, pEGFPN1-X-1b, pEGFP-N1-X-1c, pEGFP-N1-X-1d and pEGFP-N1, nontransfected cells were defined as negative control. After $48 \mathrm{~h}$, cells were analyzed by Western blot, the primary antibody is Rabbit polyclonal to GFP antibody (ab290, Abcam). No expression in negative control; GFP expression in lane 1; specific proteins expressed for four XAGE-1 splice variants; the same molecular weight protein with XAGE-1b/GFP expressed both in cells transfected pEGFP-N1-X-1a and pEGFP-N1-X-1c; lanes for transfected pEGFP-N1-X-1a, -1b, -1c and -1d plasmids have the same molecular weight protein expression with GFP.

goat anti-rabbit secondary antibody (Immunology Consultants Laboratory Inc.) 1:5000 diluted in TBS $2 \mathrm{~h}$ at room temperature. After rinsing 4 times with TBST, membrane were processed by using chemiluminescence detection system (Lot: KG136092, Thermo) according to the manufacturer's protocols.

\section{Results}

Cellular localization of four XAGE spliceosomes and their expression in COS7 cells. From testis library, we cloned the open reading frames (ORFs) of four transcripts of XAGE-1: XAGE-1a, -1b, -1c, $-1 d$ (Fig. 1A) and ligated them to the
$\mathrm{N}$-terminus of GFP. After transfecting these transcripts in COS7 cells, the cells were imaged using Leica TCS SP5 confocal microscope. Cellular localization (Fig. 2), expressed GFP protein of control and XAGE-1d/GFP fusion protein present across the cytoplasm and nucleus, while the proteins of GFP fused with XAGE-1a, $-1 \mathrm{~b},-1 \mathrm{c}$, respectively, distribute predominantly in nucleus, and they present as characteristic nuclear speckles.

Schematic representation of the four protein sequences of XAGE-1 variants shown in Fig. 1A, indicate that XAGE-1a, $-1 \mathrm{~b},-1 \mathrm{c}$ have the common sequence XAGE-1b. To detect the expression of the fusion proteins of GFP with XAGE-1 variants, we performed Western blot analysis using rabbit polyclonal to GFP antibody. The four fusion proteins were found in their corresponding molecular weight position (Fig. 3). We also detected the same molecular weight protein with XAGE-1b/GFP expressed in lanes for transfected pEGFP-N1-X-1a, pEGFP-N1-X-1c plasmids. All lanes for transfected pEGFP-N1-X-1a, -1b, -1c and -1d plasmids have the same molecular weight protein expression with GFP.

Identification of the nuclear localization signal. Considering XAGE-1a, $-1 \mathrm{~b}$ and $-1 \mathrm{c}$ share the common sequence and similar cellular localization, we thought that the NLS may be located on XAGE-1b, so, we analyzed the sequence of XAGE-1b for NLS by PSORT II program at http://psort.ims. u-tokyo.ac.jp $(13,14)$. This analysis indicates that XAGE-1b has a classical type of NLS with 4 residue pattern (PKKK) or 7 residue pattern (PKKKNQQ) at 4th amino acid (Fig. 1C). Fluoresence of deleted N-terminal 10 amino acids XAGE1b/GFP (pEGFPN1-X-1b-11F) (Fig. 1B) showed fluorescence distributed in cytoplasmic cells increased (Table I), but characteristic speckles were still similar with that of XAGE1b/GFP (Fig. 4).

Zendman et al (3) have predicted that XAGE-1b has a potential bipartite candidate NLS at amino acids 5-24. To determine whether this potential NLS is capable of directing 


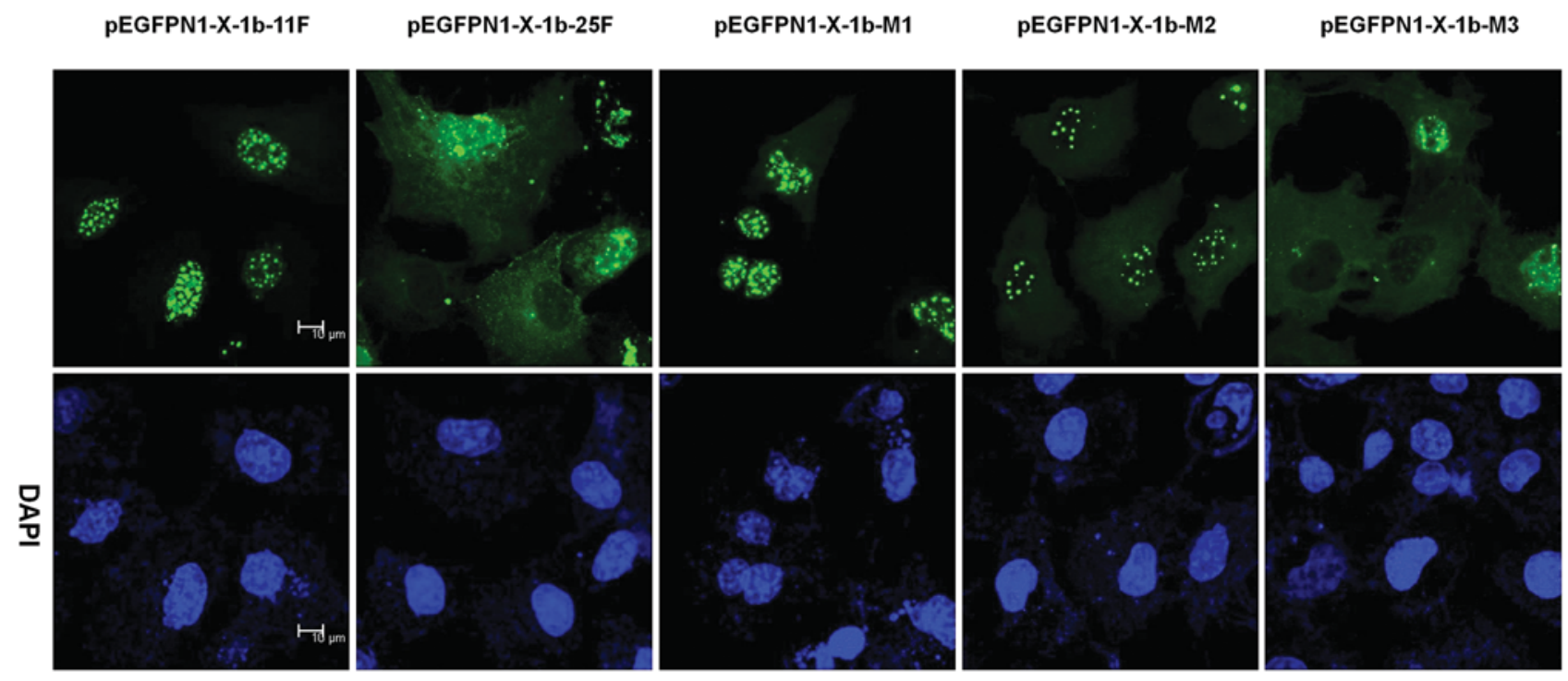

Figure 4. The bipartite NLS of XAGE-1b contributes to its nuclear localization. Fluorescence of X-1b-11F/GFP, X-1b-M1/GFP localized mainly in the nucleus, while X-1b-25F/GFP, X-1b-M3/GFP localized both in the cytoplasm and nucleus, dispersed speckles existed in some cells. Fluorescence of X-1bM2/GFP distributed in the whole cell, the characteritic speckles are still obvious. Corresponding nuclei were stained with DAPI (the lower panel). Cells were imaged using Leica TCS SP5 confocal microscope.

Table I. Quantitative scoring of fluorescent subcellular localization for constructs.

\begin{tabular}{lcc}
\hline Construct & Nuclear (\%) & $\begin{array}{c}\text { Cytosolic and } \\
\text { nuclear (\%) }\end{array}$ \\
\hline pEGFP-N1-X-1a & $71.3 \pm 2.9$ & $28.7 \pm 2.9$ \\
pEGFP-N1-X-1b & $75.7 \pm 2.9$ & $24.3 \pm 2.9$ \\
pEGFP-N1-X-1c & $73.7 \pm 3.1$ & $26.3 \pm 3.1$ \\
pEGFPN1-X-1b-11F & $55.7 \pm 5.9$ & $44.3 \pm 5.9$ \\
pEGFPN1-X-1b-M1 & $50.3 \pm 5.0$ & $49.7 \pm 5.0$ \\
pEGFPN1-X-1b-37R & $42.7 \pm 2.5$ & $57.3 \pm 2.5$ \\
\hline
\end{tabular}

COS7 cells were transfected with constructs above, fluorescent cells were counted $36 \mathrm{~h}$ after transfection by Olympic IX51 fluorescent microscope. Each value represent 3 times counts \pm s.d.

nuclear localization, we constructed further deleted mutation of the N-terminal 24 amino acids of XAGE-1b as shown in Fig. 1B, and fused these mutation forms with N-terminal of GFP (pEGFPN1-X-1b-25F). After transfection into COS7 cells, we observed that the green fluorescence localizes both in the cytoplasm and nucleus evenly, except for scattered cells still with the dispersed speckles in nucleus (Fig. 4). This distribution is very different to that of XAGE-1b.

The predicted NLS sequence include two basic domains, to verify if the different fluorescence distribution was due to these basic regions in the sequence, we performed the sitedirected mutation constructs within the NLS of XAGE-1b (Fig. 1C), as expected, the expression of $\mathrm{X}-1 \mathrm{~b}-\mathrm{M} 1 / \mathrm{GFP}(\mathrm{K} \rightarrow \mathrm{A}$ at amino acids 5-7) in COS7 cells produced still distinct dotted or speckled nuclear localization as that of pEGFPN1$\mathrm{X}-1 \mathrm{~b}-11 \mathrm{~F}$ (Fig. 4), while cells transfected with pEGFPN1-X-
1b-M3 $(\mathrm{K} \rightarrow \mathrm{A}$ at amino acids 5-7; RQKK $\rightarrow$ AQAA at amino acids 21-24) had a similar distribution with that of pEGFPN1$\mathrm{X}-1 \mathrm{~b}-25 \mathrm{~F}$. This indicates that the two basic domains in the bipartite NLS are involved in XAGE-1b nuclear localization. Fluorescence of the mutation at the second basic domain (pEGFPN1-X-1b-M2; amino acids 21-24: RQKK $\rightarrow$ AQAA) distributes both in the cytoplasm and nucleus, and the speckles are obvious.

The nuclear signal is not sufficient for the distinct nuclear localization. Although the fluorescence of GFP fusion protein with XAGE-1b-25F and XAGE-1b-M3 is distributed in the whole cell, the tiny speckles exist in the nuclei of scattered cells (Fig. 4). Aligning the sequence of XAGE-1b with that of XAGE-1d, we found that they have the N-terminal 32 amino acids in common (Fig. 1A), i.e., they all include the NLS sequence, but fused XAGE-1d/GFP protein is present both in the cytoplasm and nucleus evenly. We also ligated the N-terminal 30 amino acids of XAGE-1b (pEGFPN1-X-1b-52R) to the GFP (Fig. 1B), the fluorescence was still evenly distributed in whole cell (Fig. 5). These results indicate the nuclear signal is not sufficient for the characterized nuclear localization.

The minimum sequence for the characterized nuclear localization. To find the minimum sequence capable of directing the protein into nuleus and to form the characterized nuclear localization, we built the deletion mutation constructs of XAGE-1b from its C-terminal end (Fig. 1B). C-terminal 30 amino acids deleted mutation of XAGE-1b (pEGFPN1-X-1b-31R) showed a very small difference in fluorescence localization to that of XAGE-1b (Fig. 5); When we deleted the C-terminal 45 amino acids (pEGFPN1-X-1b-46R), the even distribution could be observed both in the nucleus and in cytoplasm. We deleted the C-terminal 39 amino acids (pEGFPN1-X-1b-40R), but 


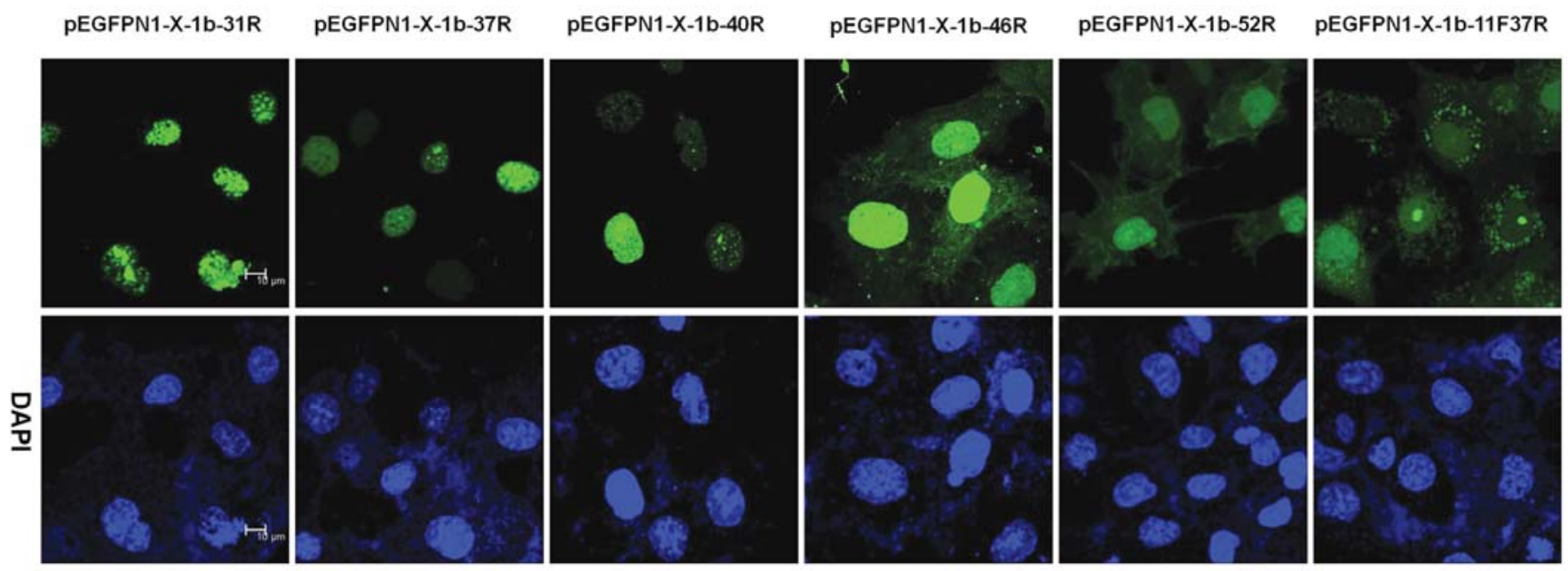

Figure 5. Possible minimum sequence for the speckled distribution of XAGE-1b in nucleus of COS7 cells. Fluorescence of X-1b-31R/GFP showed similar localization with that of X-1b/GFP; even distribution and speckled distribution in nucleus both for X-1b-37R/GFP and X-1b-40R/GFP, while X-1b-46R/GFP, $\mathrm{X}-1 \mathrm{~b}-52 \mathrm{R} / \mathrm{GFP}$ showed similar fluorescence to that of XAGE-1d/GFP. Fluorescence of X-1b-11F37R/GFP localized both in the cytoplasm and nucleus, no speckles exist in the nucleus. Corresponding nuclei were stained with DAPI (the lower panel). Cells were imaged using Leica TCS SP5 confocal microscope.

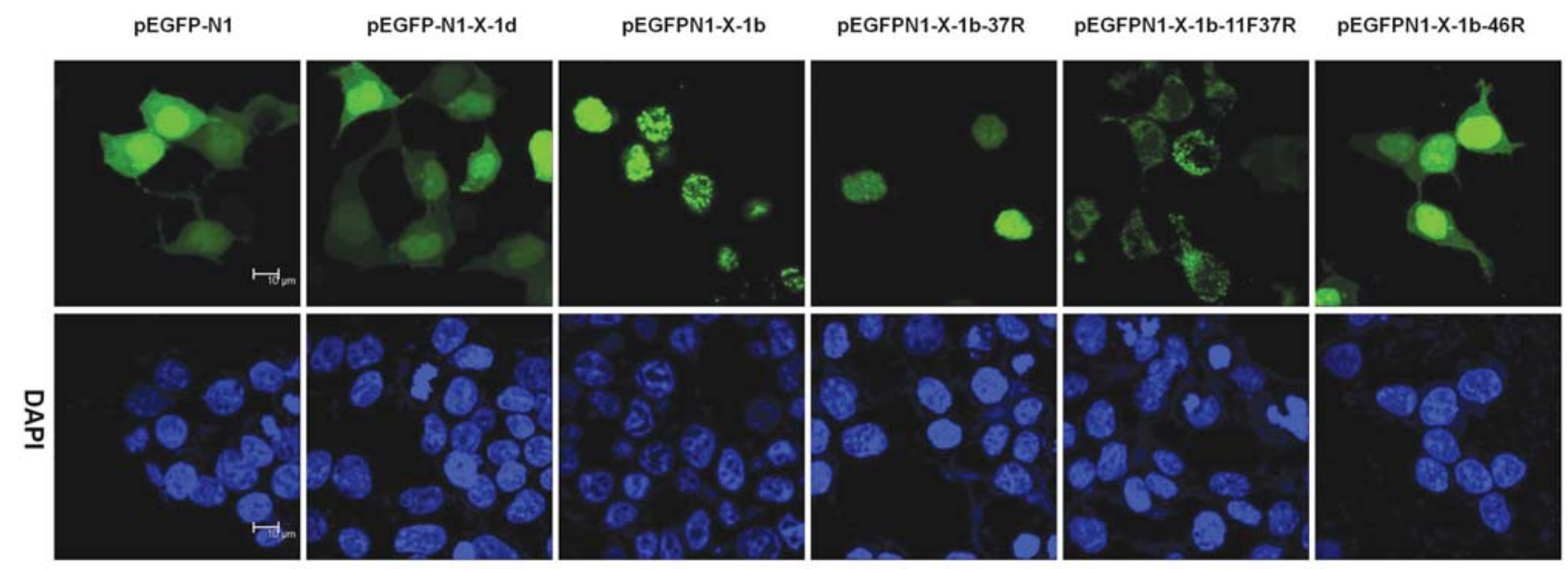

Figure 6. Possible minimum sequence for the speckled distribution of XAGE-1b in the nucleus of 293T cell. Similar fluorescence distribution as in COS7 cells. Evenly distribution and speckled distribution both exist for X-1b-37R/GFP, while X-1b-46R/GFP showed similar fluorescence to that of XAGE1d/GFP. Fluorescence of X-1b-11F37R/GFP localized both in the cytoplasm and nucleus, more increased in the cytoplasm, no speckles exist in the nucleus. Corresponding nuclei were stained with DAPI (the lower panel). Cells were imaged using Leica TCS SP5 confocal microscope.

the speckled GFP fusion protein was still observed in some COS7 cells, while in other cells, fluorescence was distributed evenly in nucleus, no speckles; so, the C-terminal 39 amino acids are not totally necessary for the speckled form.

Given that pEGFPN1-X-1b-11F showed the characteristic speckles in nucleus similar to that of XAGE-1b/GFP, we further deleted both $\mathrm{N}$-terminal 10 amino acids and $\mathrm{C}$-terminal 36 amino acids (pEGFPN1-X-1b-11F37R), transfected in cells, the GFP fusion protein still localized in the whole cell (Fig. 5); Results are more obvious in $293 \mathrm{~T}$ cells, in which fluorescence of pEGFPN1-X-b-11F37R was more expressed in the cytoplasm and slightly in the nucleus (Fig. 6). All the analysis revealed that the N-terminal 42 amino acids (may at least minimal sequence of amino acids 5-42) contribute to the characteristic dotted localization in the nucleus of XAGE-1b.

\section{Discussion}

Fourty-four CT antigens have been identified by various methods, including T-cell epitope cloning (15), SEREX (16), representational difference analysis and database mining (17). Twenty-two out of these antigens are CT-X antigens and they are usually highly expressed in the spermatogonia (4). Using spermatogenesis as a model, Hogarth et al supposed that regulation of nucleocytoplasmic shuttling may regulate gene transcription and hence cell fate (18). For the reported CT-X antigens, NY-ESO-1, SSX, SPAN-X localized both in 
the nuclei and in the cytoplasm of cells $(19,20)$, CAGE showed predominantly nuclear localization (21). Plasma cell myelomas (PMs) with pure cytoplasmic MAGE-C1/CT-7 expression showed a better prognosis than PMs with combined nuclear-cytoplasmic or nuclear expression only (22). Immunohistochemistry showed that increased cytoplasmic MAGE-A3 expression correlated with the progression of human thyroid cancer (23).

In this research, we cloned the ORFs of all four splice variants of XAGE-1 directly into pEGFP-N1, fused to the Nterminus of enhancer GFP. This is the first report on the cellular localization of all four XAGE-1 transcripts fused with GFP. Confocal microscopy showed that coupled fusion protein XAGE-1a, -1b, -1c/GFP have similar distinct speckled nuclear distribution, while XAGE-1d/GFP distribute through-out the nucleus and cytoplasm evenly. The precise different cellular localization of XAGE-1a, -1b, -1c and XAGE-1d may be related to their biological functions. Further investigation is undertaken for the different roles of the variants.

These four transcripts of $X A G E-1$ can be expressed into 146aa, 81aa, 160aa and 69aa respectively in theory, and fusion protein expressions can be verified by Western blot analysis. In previous research $X A G E-1 a$ transcript was reported translated to the XAGE-1b protein and XAGE-1C translated to 9 - and 17 -aa polypeptides $(9,12,24)$. In this study, we found the same molecular weight protein with XAGE-1b/GFP expressed both in cells transfected the pEGFP-N1-X-1a and the pEGFP-N1-X-1c plasmids. These results indicate that both the $X A G E-1 a$ transcript and XAGE1c transcript may be translated into their specific protein and XAGE-1b protein in vivo.

The active transport through the nuclear pore complex needs NLS (25). Given XAGE-1a, $-1 \mathrm{~b}$ and $-1 \mathrm{c}$ have the common sequence, XAGE-1b, we thought the NLS should be located on XAGE-1b sequence. Deleted mutation and minimal mutation demonstrated that the two basic domains at amino acids 5-24 is the bipartite NLS and it is important for the nuclear localization, this is consistent with the study of Zendman et al (3).

But NLS is not enough for the characterized nuclear distribution of XAGE-1b. The functional diameter of nuclear pore complexes (NPCs) is $9 \mathrm{~nm}$, molecules $<\sim 50 \mathrm{kDa}$ can shuttle between cytoplasm and nucleus $(25,26)$. According to this, XAGE-1a, $-1 \mathrm{~b}$ and $-1 \mathrm{c}$ should be capable of passing through the nuclear complex pore, distribute as XAGE-1d by passive diffusion; $\mathrm{N}$-terminal 24 amino acids deleted mutation still leads to dispersed speckles, although the bipartite NLS contribute to the distinct dotted distribution; and the Nterminal 24 amino acids only can not direct XAGE-1b complete into nucleus or form the distinct speckles: XAGE$1 \mathrm{~b}$ and XAGE-1d share N-terminal 32 amino acids in common, but have very different cell localization and distribution. Furthermore, deleted mutation indicates that $\mathrm{N}$ terminal 25-42 amino acids are also needed for the speckled nuclear distribution. We conclude that the N-terminal 42 amino acids (may at least amino acids 5-42) are responsible for the nuclear localization and the characterized distribution of XAGE-1b (even to XAGE-1a, -1c). This region not only has the bipartite NLS, but also may contains the sequence that have sites binding to proteins in the nucleus; or this region can form certain conformation, then binding to some protein in the nucleus and the bound protein cellular localization contributes to the speckled distribution.

Nucleus of mammalian cells is remarkably complex, it contains multiple macromolecular multiprotein complexes that have characterized morphology, called nuclear bodies (NBs). Coiled bodies are involved in snRNP biogenesis (27); PML NBs closely relate to apoptosis (28). The distinct dots of XAGE-1a, $-1 \mathrm{~b}$ and $-1 \mathrm{c}$ may belong to one of the NBs, it can be one member of the mutiprotein complexes by binding to other nuclear proteins through its $\mathrm{N}$-terminal 45 amino acids. Although the precise functions of NB remain unknown, changes in the constitution of these nuclear inclusions are associated with disease phenotypes. XAGE- $1 b$ as the main transcript of XAGE-1, and one member of CT-X gene, generally expressed in the spermatogonia (proliferating germ cells) and lung adenocarcinoma, and metastasis melanoma, may function as a transcription factor or an adaptor in the development and in tumor progress.

\section{Acknowledgements}

This work was supported by grants from the Major National Projects for Infectious Diseases (2008ZX10002-002), the National Basic Research Program of China (2006CB504304), 863 Program of China (2006AA02Z462), the Project for New Drug Development (2009ZX0913-710), the National Natural Science Foundation of China (No.30901315).

\section{References}

1. Brinkmann U, Vasmatzis G, Lee B and Pastan I: Novel genes in the PAGE and GAGE family of tumor antigens found by homology walking in the dbEST database. Cancer Res 59: 1445-1448, 1999.

2. Liu XF, Helman LJ, Yeung C, Bera TK, Lee B and Pastan I: $\mathrm{XAGE}-1$, a new gene that is frequently expressed in Ewing's sarcoma. Cancer Res 60: 4752-4755, 2000.

3. Zendman AJ, van Kraats AA, den Hollander AI, Weidle UH, Ruiter DJ and van Muijen GN: Characterization of XAGE-1b, a short major transcript of cancer/testis-associated gene XAGE-1, induced in melanoma metastasis. Int J Cancer 97: 195-204, 2002.

4. Simpson AJ, Caballero OL, Jungbluth A, Chen YT and Old LJ: Cancer/testis antigens, gametogenesis and cancer. Nat Rey Cancer 5: 615-625, 2005.

5. Scanlan MJ, Simpson AJ and Old LJ: The cancer/testis genes: review, standardization, and commentary. Cancer Immun 4: 1, 2004.

6. Van Pel A, van der Bruggen P, Coulie PG, et al: Genes coding for tumor antigens recognized by cytolytic $\mathrm{T}$ lymphocytes. Immunol Rev 145: 229-250, 1995.

7. Chomez P, De Backer O, Bertrand M, De Plaen E, Boon T and Lucas S: An overview of the MAGE gene family with the identification of all human members of the family. Cancer Res 61: 5544-5551, 2001.

8. Zendman AJ, Van Kraats AA, Weidle UH, Ruiter DJ and Van Muijen GN: The XAGE family of cancer/testis-associated genes: alignment and expression profile in normal tissues, melanoma lesions and Ewing's sarcoma. Int J Cancer 99: 361-369, 2002.

9. Egland KA, Kumar V, Duray P and Pastan I: Characterization of overlapping XAGE-1 transcripts encoding a cancer testis antigen expressed in lung, breast, and other types of cancers. Mol Cancer Ther 1: 441-450, 2002.

10. Wang T, Fan L, Watanabe Y, et al: L552S, an alternatively spliced isoform of XAGE-1, is over-expressed in lung adenocarcinoma. Oncogene 20: 7699-7709, 2001.

11. Koizumi F, Noguchi Y, Saika T, et al: XAGE-1 mRNA expression in prostate cancer and antibody response in patients. Microbiol Immunol 49: 471-476, 2005. 
12. Sato S, Noguchi Y, Ohara N, et al: Identification of XAGE-1 isoforms: predominant expression of XAGE-1b in testis and tumors. Cancer Immun 7: 5, 2007.

13. Aoyama J, Akazawa Y, Kasahara K, et al: Nuclear localization of magphinins, alternative splicing products of the human trophinin gene. J Cell Biochem 103: 765-777, 2008.

14. Nakai $\mathrm{K}$ and Horton P: PSORT: a program for detecting sorting signals in proteins and predicting their subcellular localization. Trends Biochem Sci 24: 34-36, 1999.

15. van der Bruggen $\mathrm{P}$, Traversari $\mathrm{C}$, Chomez $\mathrm{P}$, et al: $\mathrm{A}$ gene encoding an antigen recognized by cytolytic $\mathrm{T}$ lymphocytes on a human melanoma. Science 254: 1643-1647, 1991

16. Sahin U, Tureci O, Schmitt H, et al: Human neoplasms elicit multiple specific immune responses in the autologous host. Proc Natl Acad Sci USA 92: 11810-11813, 1995.

17. Scanlan MJ, Gordon CM, Williamson B, et al: Identification of cancer/testis genes by database mining and mRNA expression analysis. Int J Cancer 98: 485-492, 2002.

18. Hogarth C, Itman C, Jans DA and Loveland KL: Regulated nucleocytoplasmic transport in spermatogenesis: a driver of cellular differentiation? Bioessays 27: 1011-1025, 2005.

19. Cronwright G, Le Blanc K, Gotherstrom C, Darcy P, Ehnman M and Brodin B: Cancer/testis antigen expression in human mesenchymal stem cells: down-regulation of SSX impairs cell migration and matrix metalloproteinase 2 expression. Cancer Res 65: 2207-2215, 2005

20. Westbrook VA, Schoppee PD, Diekman AB, et al: Genomic organization, incidence, and localization of the SPAN-x family of cancer-testis antigens in melanoma tumors and cell lines. Clin Cancer Res 10: 101-112, 2004.
21. Cho B, Lim Y, Lee DY, et al: Identification and characterization of a novel cancer/testis antigen gene CAGE. Biochem Biophys Res Commun 292: 715-726, 2002.

22. Tinguely M, Jenni B, Knights A, et al: MAGE-C1/CT-7 expression in plasma cell myeloma: sub-cellular localization impacts on clinical outcome. Cancer Sci 99: 720-725, 2008.

23. Cheng S, Liu W, Mercado M, Ezzat S and Asa SL: Expression of the melanoma-associated antigen is associated with progression of human thyroid cancer. Endocr Relat Cancer 16: 455-466, 2009.

24. Nakagawa K, Noguchi Y, Uenaka A, et al: XAGE-1 expression in non-small cell lung cancer and antibody response in patients. Clin Cancer Res 11: 5496-5503, 2005.

25. Gorlich D: Transport into and out of the cell nucleus. EMBO J 17: 2721-2727, 1998

26. Kelly KF, Spring CM, Otchere AA and Daniel JM: NLSdependent nuclear localization of p120ctn is necessary to relieve Kaiso-mediated transcriptional repression. J Cell Sci 117: 2675-2686, 2004

27. Matera AG: Nuclear bodies: multifaceted subdomains of the interchromatin space. Trends Cell Biol 9: 302-309, 1999.

28. Krieghoff-Henning E and Hofmann TG: Role of nuclear bodies in apoptosis signalling. Biochim Biophys Acta 1783: 2185-2194, 2008. 